\title{
Explanation and observability of diffraction in time
}

\author{
E. Torrontegui, ${ }^{1,2}$ J. Muñoz, ${ }^{1}$ Yue Ban, ${ }^{1}$ and J. G. Muga ${ }^{1,2}$ \\ ${ }^{1}$ Departamento de Química Física, Universidad del País Vasco-Euskal Herriko Unibertsitatea, Apdo. 644, E-Bilbao, Spain \\ ${ }^{2}$ Max Planck Institute für Physik Complexer Systeme, Nöthnitzer Straße 38, D-01187 Dresden, Germany
}

(Received 18 November 2010; published 11 April 2011)

\begin{abstract}
Diffraction in time (DIT) is a fundamental phenomenon in quantum dynamics due to time-dependent obstacles and slits. It is formally analogous to diffraction of light, and is expected to play an increasing role in the design of coherent matter wave sources, as in the atom laser, to analyze time-of-flight information and emission from ultrafast pulsed excitations, and in applications of coherent matter waves in integrated atom-optical circuits. We demonstrate that DIT emerges robustly in quantum waves emitted by an exponentially decaying source and provide a simple explanation of the phenomenon, as an interference of two characteristic velocities. This allows for its controllability and optimization.
\end{abstract}

DOI: 10.1103/PhysRevA.83.043608

PACS number(s): 03.75.Be, 37.20.+j

\section{INTRODUCTION}

Diffraction in time (DIT) is a fundamental quantum dynamical effect first studied by Moshinsky [1]. One-dimensional matter waves released through a time-modulated aperture or encountering a time-dependent obstacle (for two-dimensional and three-dimensional cases, see Refs. [2,3]) show temporal quantum penumbras and interference patterns similar to the diffraction of light by spatial slits and obstacles. Understanding and controlling DIT is becoming more relevant as a result of the increasing manipulability of coherent matter waves, in particular in ultracold atomic gases and/or with ultrashort laser pulses. DIT will affect, for example, the intended applications of atom lasers [4], dynamics of matter waves emitted by ultrashort laser excitations [5], matter-wave circuits [6], and time-of-flight techniques [7]. DIT may also lead to temporal versions of diffractometers, grating spectrometry, and holography.

The original and most studied setting for DIT is the Moshinsky shutter (MS). It consists of the sudden release, by opening a shutter, of a semi-infinite plane-wave beam characterized by a "carrier" velocity. The particle density as a function of time at an observation point is formally analogous to spatial Fresnel diffraction by a sharp edge [1]. If the shutter, when closed, has reflection amplitude $R=1$, the same results are obtained from a point source with a sharp onset and constant emission thereafter [8]. Many works have applied and modified MS to study different quantum transients and, adding a potential, resonance scattering, buildup and decay, and tunneling dynamics (see, e.g., Refs. [9,10] and reviews in $[8,11]$ ). Experimentally, a DIT oscillatory pattern was first observed by Dalibard and coworkers with cold atoms falling by gravity and bouncing off a mirror consisting of evanescent light that could be switched on and off [12]. DIT through a related time-energy relation has been observed for cold neutron experiments too [7]. Interference from two time slits and time analogs of diffraction from a grating have been described for cold atoms [12,13] and ionizing atoms with ultrashort laser pulses [5]. There are also analogs of the original MS in the field of coherent transients due to frequency-chirped weak lasers [14].

DIT may be suppressed or averaged out by apodization, noise and decoherence, or unsharp carrier velocity distribu- tions $[8,15]$, so the observability of MS-DIT with matter waves has been considered a difficult task [8]. We shall see, however, that the effect is rather robust and occurs quite generally in waves emitted by an exponentially decaying resonance.

A second problematic aspect of MS-DIT is the lack of a simple and intuitive understanding of the phenomenon. The usual, geometrical "explanation" in terms of a Cornu spiral $[1,2,8]$ does not provide a simple physical picture although some insight is gained by its construction via Fresnel time zones and the Huygens principle, as in spatial diffraction [2]. Furthermore, an attempt was made in [16] to seek an explanation in terms of the Wigner distribution but, as recognized by the authors, the interpretation of the results remained ambiguous due to the lack of positivity of the Wigner function.

In this paper we address the observability and interpretation of DIT. They are linked to each other since a simple physical explanation of DIT will also provide the key to observing and controlling it. The starting point is the realization that systems that decay exponentially due to a resonance, such as cold atoms in magnetic or optical traps [17] escaping from their initial confinement, may show DIT at a distance from the trap. The density or flux oscillations will be identified as an interference effect characterized quantitatively with a simple analytical model [18]. We shall thus be able to predict and design optimal conditions for its observability, and treat on the same footing the standard constant emission after a sharp onset, and the exponentially decaying source, by modifying continuously the imaginary part of the emission pole. Figure 1, discussed later in more detail, shows the unnormalized density at an observation point away from the source. The upper curve corresponds to ordinary MS-DIT oscillations. The amplitude of the oscillations at the observation point decreases with time, and their frequency depends on time, tending to a constant. The other two curves correspond to exponentially decaying sources with different lifetimes. The oscillations are essentially the same as in the standard MS, modulated by the exponential decay.

\section{THE EXPONENTIALLY DECAYING SOURCE MODEL}

We shall use a model that was originally devised to study deviations from exponential decay [18]. We provide 


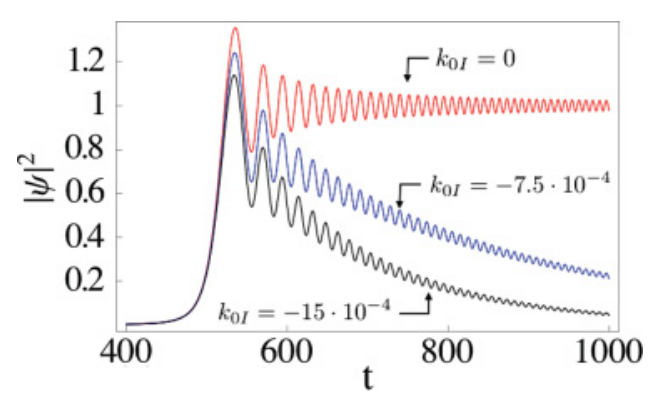

FIG. 1. (Color online) Unnormalized density versus time at $x=$ 1000 for a constant or exponentially decaying source.

here its main features. It captures the essence of resonance decay from a trap and describes analytically the external wave function without the complications and peculiarities of particular confinements. We adopt the notation in [18] with dimensionless position $x$, time $t$, and wave function $\psi$ obeying formally a Schrödinger equation for a particle of mass $1 / 2$ and $\hbar=1$,

$$
i \frac{\partial \psi(x, t)}{\partial t}=-\frac{\partial^{2} \psi(x, t)}{\partial x^{2}} .
$$

The unit of length is the inverse of the real part of the carrier wave number and the unit of time the carrier period divided by $2 \pi$. The complex dimensionless wave number $k_{0}=k_{0 R}+$ $i k_{0 I}$ and frequency of the carrier $\omega_{0}=\omega_{0 R}+i \omega_{0 I}$, obey the dispersion relation,

$$
\omega_{0}=k_{0}^{2}=\left(1+i k_{0 I}\right)^{2},
$$

so $\omega_{0 R}=1-k_{0 I}^{2}$ and $\omega_{0 I}=2 k_{0 I}$, with $k_{0 I}<0$ and $k_{0 R}=1$. The exact unnormalized solution to the Schrödinger equation for the free particle subjected to the source boundary condition $\psi(0, t)=e^{-i \omega_{0} t} \Theta(t), \quad \omega_{0 R}>0, \omega_{0 I}<0$, can be constructed by a superposition of plane waves. The resulting integral is

$$
\psi(x, t)=\frac{1}{2} e^{i k_{s}^{2} t}\left[w\left(-u_{0}^{(+)}\right)+w\left(-u_{0}^{(-)}\right)\right],
$$

where $w(z):=e^{-z^{2}} \operatorname{erfc}(-i z), \quad u_{0}^{( \pm)}= \pm(1+i) \sqrt{t / 2} k_{0}(1 \mp$ $\tau / t)$, and $k_{s}=x / 2 t, \tau=x / 2 k_{0}$ are a "saddle point" wave number and a complex characteristic time. For an observation point $x$, the saddle velocity is time dependent, $v_{s}=2 k_{s}=$ $x / t$. Figure 1 shows the unnormalized density $|\psi(x, t)|^{2}$ for different $k_{0 I}$ to illustrate the essential continuity of oscillation phenomena when varying $k_{0 I}$. If one particle is emitted, the normalized wave function is

$$
\tilde{\psi}(x, t)=\left[\int_{0}^{\infty} d t J(0, t)\right]^{-1 / 2} \psi(x, t),
$$

where $J(x, t)$ is the dimensionless flux, $J(x, t)=$ $2 \operatorname{Im}\left[\psi^{*}(x, t) \frac{\partial \psi(x, t)}{\partial x}\right]$.

\section{THE ESSENCE OF DIT}

To find the essence of DIT we may now perform an analysis technically similar to the one for tunneling times [8], with different aim, system, and results. The wave function $\psi$, for times shorter and larger than $|\tau|$ [18], can be accurately approximated by contributions from the two critical points of its defining integral, saddle, and pole,

$$
\psi(x, t)=\psi_{s}(x, t)+\psi_{0}(x, t) \Theta\left[\operatorname{Im}\left(u_{0}^{(+)}\right)\right],
$$

where the saddle $\psi_{s}$ and pole $\psi_{0}$ wave functions are defined as

$$
\begin{gathered}
\psi_{s}(x, t)=(2 t / \pi)^{1 / 2} \tau e^{i k_{s}^{2} t} /\left[(i-1) k_{0}\left(t^{2}-\tau^{2}\right)\right], \\
\psi_{0}=e^{-i \omega_{0} t} e^{i k_{0} x}
\end{gathered}
$$

Due to contour deformation along the steepest descent path from the saddle, the pole term contributes from the time when $\operatorname{Im}\left(u_{0}^{(+)}\right)=0, t_{c}=x /\left[2\left(1+k_{0 I}\right)\right]$, and decays exponentially thereafter. In a pictorial, classical association [19], the particle arriving at $(x, t)$ with velocity $v_{0}=2$ must have been released at a time $x / v_{0}$ from the source which emits particles exponentially. The saddle velocity $x / t$ is the one required for a classical particle released from $(0,0)$ to arrive at $(x, t)$. For a given $x$ each time $t$ corresponds to a different and slower saddle trajectory as time advances. Saddle trajectories may thus be pictured as the result of a burst emerging from the source with all possible velocities at $t=0$. These classical pictures are useful but, unlike long-time deviations from exponential decay [19], DIT cannot be explained by them alone. It is a quantum interference phenomenon as shown by the structure of the unnormalized density,

$$
\begin{aligned}
|\psi(x, t)|^{2}= & \left|\psi_{s}(x, t)\right|^{2}+\left|\psi_{0}(x, t)\right|^{2} \Theta\left[\operatorname{Im}\left(u_{0}^{+}\right)\right] \\
& +2 \operatorname{Re}\left[\psi_{s}(x, t) \psi_{0}^{*}(x, t)\right] \Theta\left[\operatorname{Im}\left(u_{0}^{+}\right)\right],
\end{aligned}
$$

where the asterisk denotes complex conjugation. The interference term is

$$
\begin{aligned}
2 \operatorname{Re}\left[\psi_{s} \psi_{0}^{*}\right]= & \sqrt{\frac{t}{\pi}} \frac{2 \beta(x, t)}{16\left|\omega_{0}\right|^{2} t^{4}+x^{4}-8 t^{2} x^{2} \omega_{0 R}} \\
& \times\left[\left(8 \omega_{0 R} x t^{2}-2 x^{3}\right) \cos \phi+8 \omega_{0 I} x t^{2} \sin \phi\right],
\end{aligned}
$$

where $\quad \phi(x, t)=\left(\omega_{0 R}+k_{s}^{2}\right) t-x-\frac{3 \pi}{4}, \quad$ and $\quad \beta(x, t)=$ $e^{\omega_{0 I} t-k_{0 I} x}$. Light does not show DIT in vacuum because there is no dispersion and no interference of this kind.

Figure 2 shows the agreement at times larger and shorter than $|\tau|$ between exact and approximate wave functions. The pole and saddle terms separately do not oscillate in time,

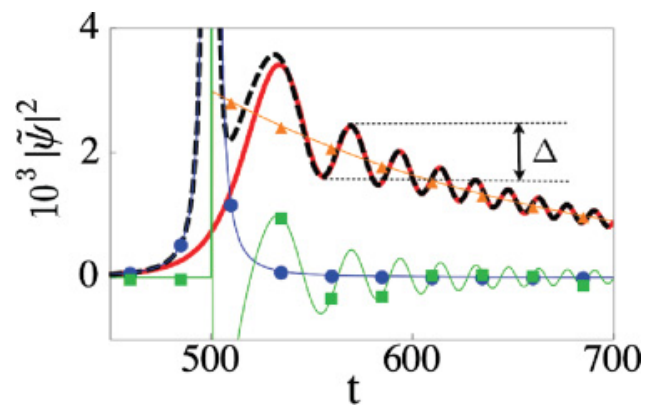

FIG. 2. (Color online) Normalized probability densities versus time for $k_{0}=1-0.0015 i$ at $x=1000$. Exact (red solid line), approximate [Eq. (7); black dashed line], saddle term (blue circles), pole term (orange triangles), and interference term [Eq. (8); green squares]. 
whereas the interference term, Eq. (8), reproduces accurately the DIT oscillations.

\section{CHARACTERIZATION AND OBSERVABILITY OF DIT}

The frequency of the DIT oscillation depends on the interference of the saddle and pole frequencies $k_{s}^{2}$ and $\omega_{0 R}$ and, as $k_{s}$ depends on time, the DIT oscillation period is not constant. From Eq. (8) we can infer the position of the $n$th maximum. For $\left|k_{0 I}\right| \ll 1$, the $\sin \phi$ term of Eq. (8) tends to vanish so the DIT oscillations are essentially due to the $\cos \phi$ term. The maxima correspond to $\phi\left(x, T_{n}\right)=2 n \pi$ at times,

$$
T_{n}=\frac{(3+8 n) \pi+4 x+\sqrt{[(3+8 n) \pi+4 x]^{2}-16 \omega_{0 R} x^{2}}}{8 \omega_{0 R}},
$$

where $n=0,1,2, \ldots$ ( $n=0$ is for the principal maximum). The interval $T_{n+1, n} \equiv T_{n+1}-T_{n}$ between two consecutive maxima is in good agreement with the exact, numerically calculated period (see Fig. 3). The small discrepancy at $n=0$ can be attributed to the dependence on time of the factors multiplying $\cos \phi$ and the proximity of $|\tau|$.

For large times the period of the DIT oscillations tends to the carrier period, $\lim _{n \rightarrow \infty} T_{n+1, n}=2 \pi / \omega_{0 R}$. The amplitude of the oscillations decays relatively slowly compared to the pole term, as $e^{\omega_{01} t} t^{-3 / 2}$ [see Eq. (8)], but exponentially faster than the saddle term.

According to Eq. (9), $T_{0}$ is not a linear function of $x$. For example, in the limit $k_{0 I} \rightarrow 0$ the motion of the first maximum is described by $x_{0}=2 T_{0}-\sqrt{3 T_{0} \pi}$. Even though an asymptotic velocity may be defined, $2\left(1+k_{0 I}\right)$ in the general case (see the inset of Fig. 3), there is no oblique asymptote for this function. Thus a naive linear extrapolation back to the origin at some large distance fails to provide the instant of the source onset. In other words, the times in which the tangents to $x_{0}(t)$ cut $x_{0}=0$ have no definite limit, in spite of the well-defined asymptotic velocity. This is an example of the importance of DIT to correct simple classicaldynamical extrapolation from asymptotic wave features to extract emission characteristics, as practiced, for example, in the analysis of ionization by ultrashort laser pulses [20].

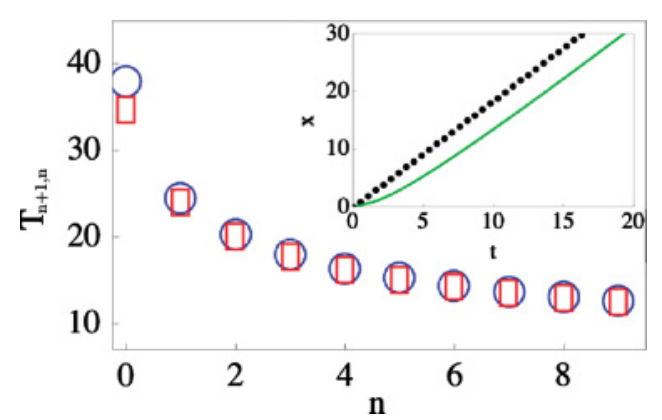

FIG. 3. (Color online) Time intervals $T_{n+1}-T_{n}$ between two consecutive maxima: exact (circles), and approximation from Eq. (9) (boxes). Same parameters as in Fig. 2. The symbol size is to help the eye, and is not related to errors. (Inset) Nonlinear position of the first maximum versus time for $k_{0 I}=-0.08$ (solid line) and onset of the pole term $x_{c}=2\left(1+k_{0 I}\right) t$ (dotted line).

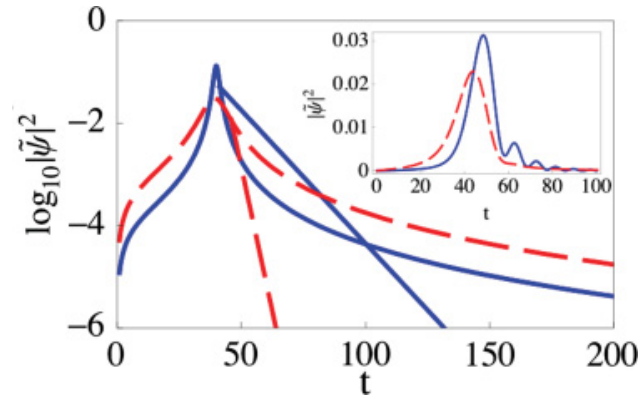

FIG. 4. (Color online) Saddle and pole terms for $k_{0 I}=-0.03$ (solid blue line) and $k_{0 I}=-0.13$ (long-dashed red line). $x=80$. The inset shows the corresponding exact densities. The shorter lifetime suppresses DIT.

In our dimensionless description two factors affect the visibility of the DIT pattern: the observation position $x$ and the lifetime. Figure 4 shows the moduli of the logarithm of the pole and saddle densities for two different lifetimes. The pole term is a semi-infinite straight line which begins when the pole is crossed by the steepest descent path passing along the saddle in the complex momentum plane, at $t_{c}$; the saddle term shows a maximum near $|\tau|$ and decays from there slowly. There may be up to two intersections of the two terms, one near the arrival of the main front, and one at a long time that marks the transition to postexponential decay [18]. When the saddle and pole terms are similar or close enough, the interference oscillations appear. The interference region which interests us here is the one following the main front because it relates by continuity to ordinary MS-DIT in the limit $k_{0 I} \rightarrow 0$; it is also much more easily observable than the oscillations at large times because of the magnitude of the amplitudes.

The oscillations are evidently not present at the source $x=0$, and will be small at small distances, $x \lesssim 1$, because of the rapid decay and separation from the pole term of the saddle term in these conditions (see Fig. 5). The saddle term beyond the main front arrival increases with $x$ [18]. In the opposite extreme of very large $x$, it eventually dominates entirely and stays above the pole term at all times, suppressing DIT and even exponential decay [18]. Between these two extreme scenarios DIT is prominent in an ample range of $x$. The slope of the pole term also plays a role. For larger values (smaller lifetimes), pole and saddle contributions separate more rapidly leading to fewer visible DIT oscillations which may actually disappear for small enough lifetimes.

To estimate the domain where some oscillations are seen before the decay is too strong we may solve $T_{1,0}<N \tau_{0}$ for

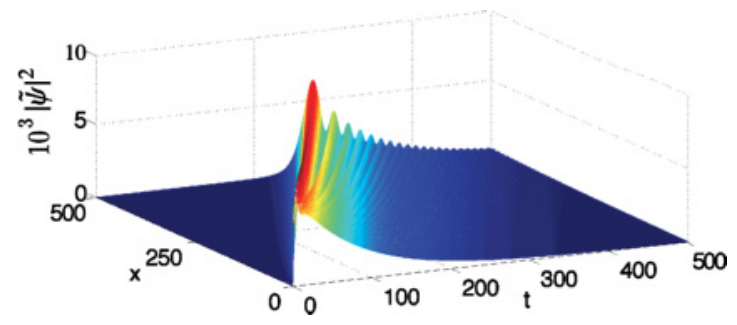

FIG. 5. (Color online) Density $|\tilde{\psi}(x, t)|^{2}$ for $k_{0 I}=-0.003$ showing the transition from pure exponential decay to a DIT pattern. 
a small $N$, where $\tau_{0}=1 / 4\left|k_{0 I}\right|$ is the lifetime. This gives an explicit but lengthy expression. For $N=5$ and in the $k_{0 I} \rightarrow 0$ limit, $x \lesssim 30 \tau_{0}^{2}$.

From the previous discussion it might seem that a very long lifetime is always preferable to attain DIT. Nevertheless long lifetimes also imply a weaker signal because of the normalization. The consequence of opposite tendencies is an optimal lifetime-position point. A good measure of the visibility of DIT of experimental relevance is the difference $\Delta$ between the second maximum and the previous minimum of the normalized probability density (see Fig. 2). The optimal parameters are found to be $k_{0 I}=-0.03, x=60$.

\section{MODEL INDEPENDENCE OF THE RESULTS}

We have described the close connection between DIT and resonance decay. DIT will be visible when contributions from different resonances are well separated, which generally requires narrow and/or strong confinement. DIT does not depend on the specific properties of the model used so far. We have checked the robustness of DIT from exponential decay explicitly with several additional models. Winter's decay model [21] describes the decay of the ground state of the square well between $-L$ and 0 when the right infinite wall is substituted by a $U \delta(x)$ potential. The wave function outside the trap tends to the source model wave function for large $U$ [22]. Moreover, DIT does not depend dramatically on the strict confinement of the initial wave function on a finite domain. To show this we have calculated the decay of the ground state of a well with a finite right wall when the right wall is substituted by a delta barrier. The initial wave penetrates by tunneling in the evanescent region of the right wall and this creates a different fast forerunner at $x$, but the part associated with the dominant, lowest energy resonance remains essentially stable showing DIT as for the infinite wall (see Fig. 6).

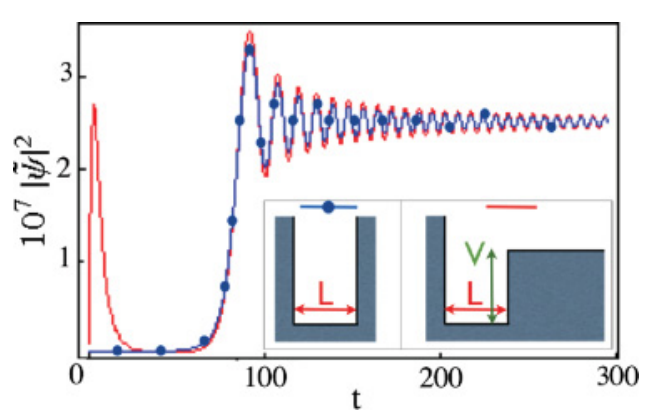

FIG. 6. (Color online) Stability of DIT for decay from $U \delta(x)$ (Winter's model). The initial states are the ground states of the two wells of the inset. At $t=0$ the right wall is substituted by the delta. $L=3.14, x=157.05, U=161.35, V=202.72$.

Moreover, we have observed the same stability for finite-width barriers. DIT also survives a smooth source onset [4], and again may be observed after the passage of some onset-dependent transients. As for the effect of collisions, in the mean-field regime DIT is enhanced for attractive interactions [8].

Let us finally point out the possibility of observing DIT in periodic structures [9] such as optical lattices, or other physical systems that realize a tight-binding model, for example, periodic waveguide arrays that provide a classical, electric field analog of a quantum system with exponential decay [23].

\section{ACKNOWLEDGMENTS}

We thank A. del Campo, D. Guéry-Odelin, J. Martorell, D. Sprung, and E. Sherman for discussions. We acknowledge funding from the Basque Government (Grant No. IT472-10) and Ministerio de Ciencia e Innovación (Grant No. FIS200912773-C02-01). Y.B. and E.T. acknowledge financial support from the Basque Government (Grants No. BFI-2010-255 and No. BFI-08-151).
[1] M. Moshinsky, Phys. Rev. 88, 625 (1952).

[2] C. Brukner and A. Zeilinger, Phys. Rev. A 56, 3804 (1997).

[3] S. Godoy, Phys. Rev. A 67, 012102 (2003).

[4] A. del Campo, J. G. Muga, and M. Moshinsky, J. Phys. B 40, 975 (2007).

[5] G. G. Paulus and D. Bauer, Lect. Notes Phys. 789, 303 (2009).

[6] D. Schneble, M. Hasuo, T. Anker, T. Pfau, and J. Mlynek, J. Opt. Soc. Am. B 20, 648 (2003).

[7] Th. Hils, J. Felber, R. Gahler, W. Glaser, R. Golub, K. Habicht, and P. Wille, Phys. Rev. A 58, 4784 (1998).

[8] A. del Campo, G. García-Calderón, and J. G. Muga, Phys. Rep. 476, 1 (2009).

[9] G. Monsivais, M. Moshinsky, and G. Loyola, Phys. Scr. 54, 216 (1996).

[10] G. García-Calderón and J. Villavicencio, Phys. Rev. A 64, 012107 (2001).

[11] M. Kleber, Phys. Rep. 236, 331 (1994).

[12] P. Szriftgiser, D. Guéry-Odelin, M. Arndt, and J. Dalibard, Phys. Rev. Lett. 77, 4 (1996).
[13] Y. Colombe, B. Mercier, H. Perrin, and V. Lorent, Phys. Rev. A 72, 061601 (2005).

[14] S. Zamith, J. Degert, S. Stock, B. deBeauvoir, V. Blanchet, M. A. Bouchene, and B. Girard, Phys. Rev. Lett. 87, 033001 (2001).

[15] S. Godoy, Physica B 404, 1826 (2009).

[16] V. Man, M. Moshinsky, and A. Sharma, Phys. Rev. A 59, 1809 (1999).

[17] S. R. Wikinson et al., Nature (London) 387, 575 (1997).

[18] E. Torrontegui, J. G. Muga, J. Martorell, and D. W. L. Sprung, Phys. Rev. A 80, 012703 (2009).

[19] E. Torrontegui, J. G. Muga, J. Martorell, and D. W. L. Sprung, Phys. Rev. A 81, 042714 (2010).

[20] Y. Ban, E. Y. Sherman, J. G. Muga and M. Bütiker, Phys. Rev. A 82, 062121 (2010).

[21] R. G. Winter, Phys. Rev. 123, 1503 (1961).

[22] E. Torrontegui, J. G. Muga, J. Martorell, and D. W. L. Sprung, Adv. Quant. Chem. 60, 485 (2010).

[23] G. Della Valle, S. Longhi, P. Laporta, P. Biagioni, L. Dou, and M. Finazzi, Appl. Phys. Lett. 90, 261118 (2007). 